Classification

Physics Abstracts

$61.30 \mathrm{E}-61.40 \mathrm{~K}$

\title{
Phase separations in the mixtures of a mesomorphic polymer with a low molecular mass liquid crystal
}

\author{
C. Casagrande, M. Veyssié and H. Finkelmann $\left({ }^{*}\right)$ \\ Physique de la Matière Condensée, Collège de France, \\ 11, place Marcelin-Berthelot, 75231 Paris Cedex 05, France \\ (*) Institut für Physikalische Chemie, 3392 Clausthal-Zellerfeld, R.F.A.
}

(Recu le 27 mai 1982, accepté le 23 juillet 1982)

\begin{abstract}
Résumé. - Nous avons étudié le diagramme de phase d'un système binaire constitué d'un polymère mésomorphe à chaîne latérale et d'un monomère analogue aux groupes mésogènes du polymère. Nous montrons en particulier qu'il n'y a pas miscibilité totale de ces composés; ce qui conduit à l'apparition de deux phases nématiques en coexistence à une même température.

Abstract. - We have studied the phase diagram of a binary system, constituted with a side-chain mesomorphic polymer and a monomer liquid crystal, very similar to the mesogenic groups in the polymer. The main results is the existence of a gap of miscibility in the nematic state, leading to the coexistence, at a same temperature, of two nematic phases.
\end{abstract}

1. Introduction. - During these last years, an increasing interest has been devoted to thermotropic mesomorphic polymers, either they have mesogenic groups in the main chain [1], or in side chains [2]; this interest is justified both by the eventual technological applications of these materials and by the fundamental problems they arise. A review on the subject can be found in reference [3] and [4]. One possible approach to get information on these polymers is to determine the influence of such macromolecules when solubilized in conventional liquid crystals; this method appeared rather fruitful in the case of flexible chains in nematic solvent that we recently studied [5].

In a first step, we were thus led to determine the phase diagram of a binary mixture, constituted by a side chain nematic polymer with a low molecular mass liquid crystal, similar to the mesogenic groups in the macromolecule. What should have been a simple control measurement turned out to reveal a rather complex phase diagram, which, in particular, puts into evidence the coexistence of two non-miscible nematic phases. In this paper, after a rapid description of the experimental methods we used, we shall present and discuss our results, and compare them to previously reported data for analogous compounds [6].

2. Experimental section. - 2.1 MATERIALS AND mixtures. - The formulaes and the transition temperatures of the two pure compounds are given in table $I$. The polymer $(P)$ is a 
Table I.

\begin{tabular}{|c|c|c|c|}
\hline & Formulaes & $\begin{array}{l}\text { Phase } \\
\text { tramitions }\end{array}$ & Ref. \\
\hline$P$ & 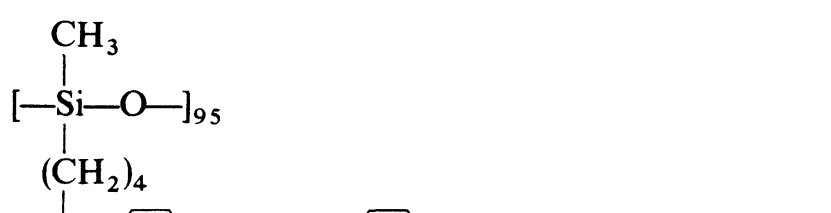 & $\underset{376 \mathrm{~K}}{\longrightarrow} \mathrm{I}$ & This work \\
\hline M & $\mathrm{H}_{13} \mathrm{C}_{6} \mathrm{O}-\left\langle\bigcirc-\mathrm{COO}-\mathrm{O}-\mathrm{OC}_{8} \mathrm{H}_{17}\right.$ & $\mathrm{S}_{\mathrm{c}} \underset{38 \mathrm{~K}}{\longrightarrow} \mathrm{N} \underset{365 \mathrm{~K}}{\longrightarrow} \mathrm{I}$ & This work \\
\hline $\mathbf{M}_{1}$ & $\mathrm{H}_{13} \mathrm{C}_{6} \mathrm{O}-\mathrm{O}-\mathrm{COO}-\mathrm{O}-\mathrm{OCH}_{2}-\mathrm{CH}=\mathrm{CH}_{2}$ & $\underset{360 \mathrm{~K}}{\mathrm{~N}} \mathrm{I}$ & [6] \\
\hline
\end{tabular}

poly-dimethyl-siloxane with a phenylbenzoate in the side chain, connected to the main chain by a flexible spacer of four methylene groups; the average degree of polymerization is 95 (molar mass $\simeq 35000$ ). It presents a nematic to isotropic state transition at $376 \mathrm{~K}$. The low molecular mass compound (M) is also a phenylbenzoate; it exhibits a smectic $C$ and a nematic phase. Mixtures with different concentrations are prepared either by solubilization in chloroform and evaporation under vacuum, or by direct mixing; in both cases, it appears necessary to homogenize the samples, kept in glass tube sealed under primary vacuum, by smooth shaking in the isotropic phase, during periods of the order of several hours. The indicated concentration, $x$, refers to the weight fraction of the polymer $(\mathrm{P})$ in $(\mathrm{M})$.

2.2 Procedure. - For the determination of the phase diagram we used three different techniques, which give complementary informations.

a) Direct observation under polarizing microscope;

b) Visual observation in sealed tubes which are immerged in a thermostated oil bath; the bath temperature is regulated with an accuracy of $\pm 0.1 \mathrm{~K}$;

c) Differential scanning calorimetry, using a DSC 2 Perkin Elmer apparatus at slow heating and cooling rate $(5 \mathrm{~K} / \mathrm{min}$. or $2.5 \mathrm{~K} / \mathrm{min}$.).

By method $a$ ) and, more precisely by method $b$ ), we can detect the phase separations when they occur, and determine the extension of the biphasic domains.

As discussed in details later, the main feature of the system is a pronounced tendency to phase separation in the nematic state. We have to underline that the kinetics of this process is very slow, as well as the rehomogenization rates after a phase separation. This implies a great care in the control of the thermal history of the samples, and on an appropriate choice for the cooling and heating rate, in order to be close to the thermodynamical equilibrium.

3. Experimental results. - Using method $b$ ), we obtain reproducible and consistent results as operating as follows : a mixture, with concentration $c$, is homogenized at $T \simeq 383 \mathrm{~K}$, and dipped in the thermostated bath at the same temperature. We slowly cool the bath, with a rate of $0.1 \mathrm{~K} / \mathrm{min}$, and detect the temperatures where some heterogeneity appears in the sample (cf. Fig. 1) the observed phenomena depend on the initial concentration. 


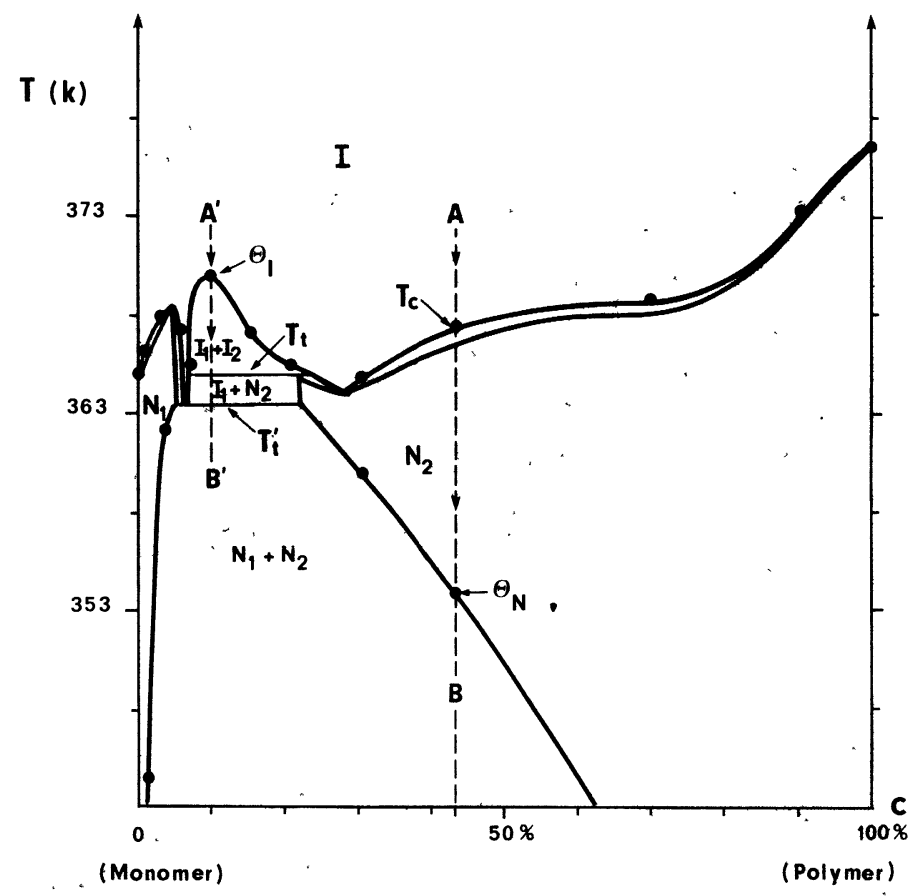

Fig. 1. - Phase diagram of the monomer-polymer system ; $c$ is the weight fraction of $(P)$ in $(M) . I=$ isotropic phase. $\mathrm{N}=$ nematic phase.

$3.1 c<6 \%$ AND $c>23 \%$ - Starting from a point such A (cf. Fig. 1), the isotropic phase remains homogeneous, till a temperature $T_{\mathrm{c}}$ where the first nematic droplets appear, the diphasic isotropic-nematic domain is rather narrow $(\simeq 0.5$ and $1.5 \mathrm{~K})$, then we observe an homogeneous nematic phase. When cooling further, it appears a new phase separation at $\theta_{\mathrm{N}}$, with a meniscus delimitating two turbid birefringent phases; by observation under polarizing microscope, we identify the two subphases as nematic ones. We conclude that this is a phase separation process, with coexistence of two nematic states $\mathrm{N}_{1}$ and $\mathrm{N}_{2}$.

We emphasize, once more, the slowness of the kinetics of the phase separation and, moreover, of the re-mixing proeess : for instance, if we heat a sample after the $\mathrm{N}_{1}-\mathrm{N}_{2}$ separation has occurred, a meniscus remains even in the isotropic phase; the mixture becomes homogeneous only after shaking at this temperature.

The main features of this part of the phase diagram are confirmed by DSC measurements : starting with a room temperature sample with concentration $c$, we observe no peak at the $\theta_{\mathrm{N}}$ temperature, excluding possible first order transition. Near $T_{\mathfrak{c}}$, two peaks appear, the corresponding enthalpy being of the same order of magnitude as for pure compound at the $\mathrm{N} \rightarrow \mathrm{I}$ transition; after annealing in the isotropic state, just one peak subsists at the $\mathrm{I} \rightarrow \mathrm{N}$ transition when cooling again. This can be explained if we suppose that the thermodynamic equilibrium is not reached when heating, in the DSC apparatus, even at a slow rate : a partial phase separation subsists above $\theta_{\mathrm{N}}$, with subphases having slightly different $T_{\mathrm{c}}$ values; this leads to the appearance of two distinct peaks. This splitting disappears when the sample is homogenized by annealing above $T_{\mathrm{c}}$.

Notice also that, at very low concentration, a nematic $\rightarrow$ smectic $C$ transition is detected by visual observation, which correspond to a weaker peak in the DSC data.

Careful observations under polarizing microscope, on free surface droplets, reveal that a periodic pattern appears, when cooling, near the $\theta_{\mathbf{N}}$ value (Fig. 2); at lower temperature, this pattern vanishes while a finely dispersed heterogeneous system appears. It is reasonable to think 


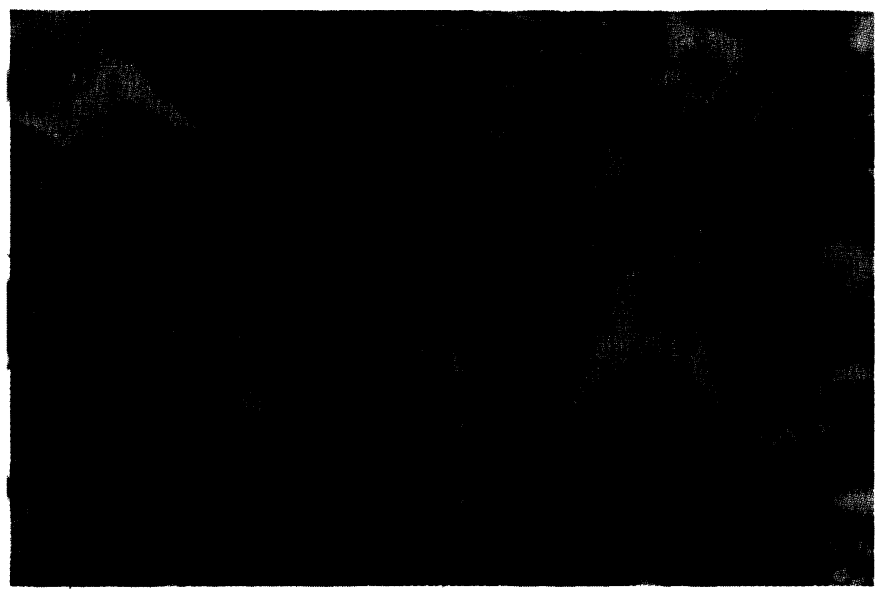

a)

Scale : $\mapsto$

$10 \mu \mathrm{m}$

b)

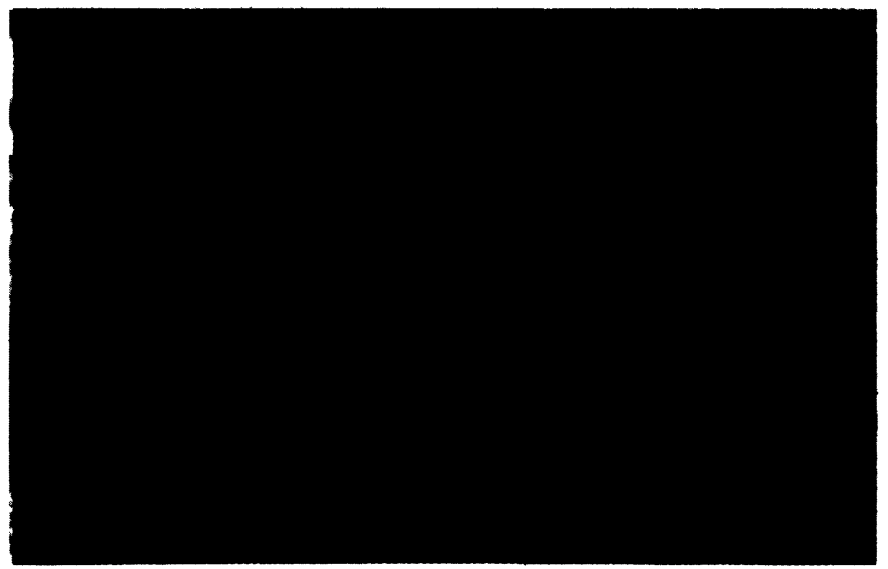

c)

Fig. 2. - Evolution of the appearance of a free droplet observed under polarizing microscope when the temperature is decreased $(\bar{c}=30 \%)$. a) $\left.\left.T>\theta_{\mathrm{N}}, b\right) T \simeq \theta_{\mathrm{N}}, c\right) T<\theta_{\mathrm{N}}$. 
that this periodic pattern is related to a fluctuation of concentration when passing through the phase separation curve. Further investigation is necessary to determine wether it is mainly a bulk or a surface effect; but it is perhaps an interesting way to study the initiation of spinodal decomposition in polymer solutions, the visualization of this effect being facilitated by the birefringence of these mesophases.

$3.26 \%<c<23 \%$ - In this region, the situation is somewhat complex, and more delicate to elucidate. Decreasing the temperature from the point $\mathrm{A}^{\prime}$, a slight turbidity, appears at $\theta_{\mathrm{I}}$; the first nematic droplets are observed only at a lower temperature, $T_{t}$, which is the same for all the mixtures in the concentration range. At still lower temperature $\left(T<T_{t}^{\prime}\right)$, the sample is totally nematic, but presents two separated subphases. These results imply a phase separation in the isotropic state, and the existence of two triple points, $T_{\mathrm{t}}$ and $T_{t}^{\prime}$, very close together $\left(T_{\mathrm{t}}=365 \mathrm{~K}, T_{\mathrm{t}}^{\prime}=363.5 \mathrm{~K}\right)$.

4. Conclusion. - The most striking result, in this polymer-monomer system, is the existence of a large gap of miscibility in the nematic state, delimitated by a curve which exhibits an asymmetrical shape. This situation is classical in conventional solutions of polymers below the Flory temperature. It appears somewhat unusual in the liquid crystal field : for the extensively studied low molecular liquid crystals, and even for the more recent examples of polymer-monomer mixtures $[6,7,8]$, one observes, in general, total miscibility in the nematic phase. However, we emphasize that the present results are quite compatible with the miscibility rule, stating that, if two compounds present a total miscibility, they are isomorphic, while the reverse proposition is not true.

Another interesting point results from the comparison between the present system and a very similar one recently studied [6] : with the same polymer $P$, and a slightly different monomer $M_{1}$ (cf. Table I), a much more simple phase diagram is abserved, presenting a total miscibility in the nematic range $\left({ }^{1}\right)$. This points out that these orientated solutions are very sensitive to the details of the molecular structure; we may infer that the presence of the siloxane chain impose drastic steric conditions which have to be fitted by the monomer liquid crystal, compared to the side chain groups. Further work is currently underway to specify these compatibility conditions.

Acknowledgments. - We have benefited from discussions with L. Auvray, J. Billard, F. Brochard and C. Noël, and from considerable help from A. Collet and J. Brienne in DSC measurements.

$\left({ }^{1}\right)$ Recent preliminary studies on another compound which does not present a smectic phase also revealed a miscibility gap in the nematic state. This seems to answer the question raised by the referee and rules out a correlation between the $\mathrm{N}_{1}-\mathrm{N}_{2}$ separation and the existence of a smectic $C$ phase for the monomer $M$ via pretransitional cybotactic groups.

\section{References}

[1] Roviello, A. and Sirigu, A., J. Pol. Sci. Pol. Let. Ed. 13 (1975) 455.

[2] Finkelmann, H., Ringsdorf, H. and Wendorff, J. H., Makromol. Chem. 174 (1978) 273.

[3] Blumstein, A. and Hsu, E. C., in Liquid Crystalline Order in Polymer A. Blumstein Ed. (Academic Press), 1978, p. 105.

[4] Polymer Liquid Crystals, A. Cifferi, W. R. Kyhan and R. B. Meyer Ed. (Academic Press New York) (to be published).

[5] De Gennes, P. G. and Veyssié, M., Structural order in Polymers, F. Ciardeli and P. Giusti Ed. (Pergamon Press Oxford and New York) 1981;

Dubault, A., Thesis, Paris VI University (1981).

[6] Finkelmann, H., Kock, H. J. and Rehage, G., Mol. Cryst. Liq. Cryst. (to be published).

[7] Millaud, B., Thiery, A., Skoulios, A., Mol. Cryst. Liq. Cryst. Lett. 41 (1978) 263.

[8] Nö̈l, C., Billard, J., Mol. Cryst. Liq. Cryst. Lett. 41 (1978) 269.

[9] Griffin, A. C., Hauens, S. J., Mol. Cryst. Liq. Cryst. Lett. 49 (1979) 239. 